\title{
A Secure Simultaneous Wireless Information and Power Transmission System Based on CDMA Architecture
}

\author{
F. Zhao, Y.T. Wu, Y.Z. Sun \\ The Key Laboratory of Specialty Fiber Optics and Optical Access Networks \\ Shanghai University
}

\begin{abstract}
In view of the security issue of the simultaneous wireless information and power transmission (S WIPT), this paper presents a secrecy S WIPT communication system based on code division multiple access (CDMA) architecture. The transmitter uses different spread spectrum polynomials to spread spectrum information and superimposes a narrow band energy signal. Then the transmitter sends information and energy to the legitimate user terminals. Each legitimate user terminal collects energy and despread the received information by using its own spread spectrum polynomial. Although the eavesdroppers can receive all the information sent by the transmitter, they are unable to correctly despread or decrypt information without spreading polynomials of legitimate users. Secure information transmission is thereby achieved. Moreover, a modified adaptive allocation algorithm of orthogonal-variable-spreading-factor (OVSF) code is proposed for the new system.
\end{abstract}

Keywords-SWIPT; generated polynomial; OVSF code

\section{INTRODUCTION}

Simultaneous wireless information and power transfer (SWIPT) refers to using the same emitted electromagnetic wave field to transport both energy that is harvested at the receiver, and information that is decoded by the receiver. Seeking new types of energy source to prolong the lifetime of the energy-constrained wireless network becomes a spotlight.

In [1], Zhang and Ho proposed two SWIPT schemes, where the former allocates different time blocks for ID and EH, whereas the latter splits the signal into two streams for ID and EH. In [2], a dynamic power splitting approach for the SWIPT in SISO and MISO systems was proposed. In [3-5], the power splitting SWIPT scheme in orthogonal frequency division mu ltiple access (OFDMA) systems was investigated. Owing to the openness of the wireless transmission medium, wireless information is susceptible to eavesdropping. Thus, secure communication is a critical issue for the SWIPT. The secure beam forming schemes for the SWIPT in a MISO broadcast channel were studied in $[6,7]$. In [8], the power allocation problem to maximize the achievable secrecy rate in a downlink OFDMA system was studied. However, the channel from the transmitter to the eavesdropper is assumed to be known in [8], which cannot be achieved in practical situations.

In this paper, we propose a new secrecy SWIPT transmission system based on CDMA architecture. The transmitter is assumed to know the spread spectrum sequence generating polynomial of each legitimate user terminal, which is unique for each user. The legitimate user terminal only know their own generation polynomial, an eavesdropper doesn't know polynomial of legitimate user terminal. The transmitter encrypts or spreads the information which is sent to the legitimate user terminal. Each legitimate user terminal can dispread the received information by its polynomial, while it is unable to decrypt or dispread other legitimate user terminal's information. Since the eavesdropper has no knowledge of the generated polynomial of legitimate users, it cannot correctly dispread or decrypt information. Therefore, a secure communication is achieved. As the transmitter need more channels than the legitimate receiver and the OVSF code numbers are limited, the downlink OVSF code allocation strategy plays a key role, which directly determines the capacity of the system.

\section{SYSTEM MODEL}

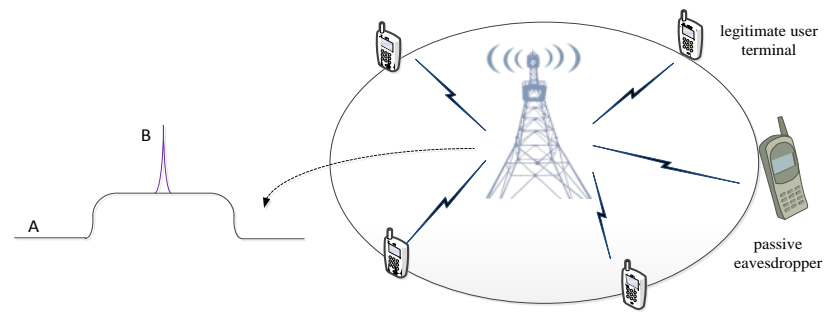

FIGURE I. THEPROPOSED MULTIUSER SWIPT SYSTEM MODEL

Consider a downlink CDMA SWIPT system shown in Figure 1. The system is composed of a transmitter, $\mathrm{K}$ legitimate receivers and an eavesdropper. Transmitter signal is a composite signal $\mathrm{A}+\mathrm{B}$, which is the superposition of a wide band signal and a narrow band signal. The wide band signal A is a CDMA spread spectrum signal. It is used to send information to the legitimate user terminal. The narrow band signal $\mathrm{B}$ is a narrow signal which has very strong energy. It can be used for a legitimate us er terminal charge.

Wideband signal $\mathrm{A}$ is a multiple access CDMA signals, which is a superposition of $\mathrm{K}$ signals with the same frequency, the same time and different codes. Due to using address code to distinguish users, user signals have no restrictions on the frequency, time and space. The working principle diagram of the system normal communication is shown in figure 2 . 


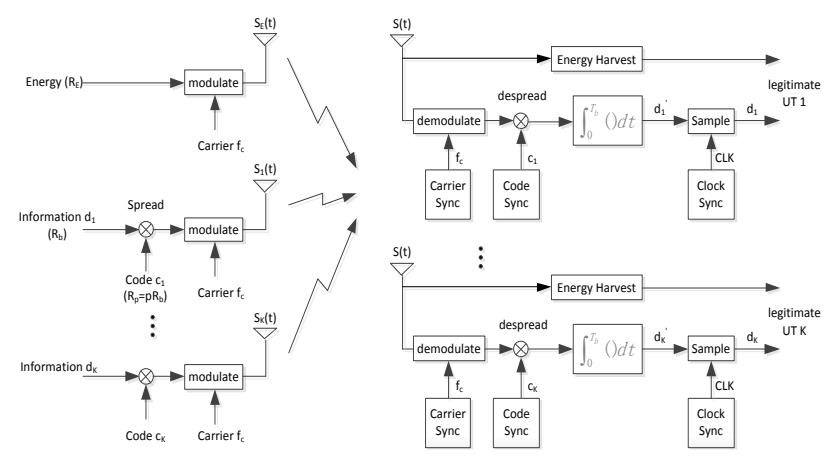

FIGURE II. THE WORKINGPRINCIPLE DIAGRAM OF SWIPT SYSTEM

$$
\begin{gathered}
\mathrm{S}(\mathrm{t})=\sum_{\mathrm{k}=1}^{4} \sqrt{2 \mathrm{P}_{\mathrm{C}_{\mathrm{k}}}} \mathrm{D}_{\mathrm{k}}(\mathrm{t}) \mathrm{x}_{\mathrm{k}}(\mathrm{t}) \sin \left(2 \pi \mathrm{f}_{\mathrm{c}}+\theta_{\mathrm{k}}\right)+ \\
\sqrt{2 \mathrm{P}_{\mathrm{C}_{\mathrm{E}}}} \sin \left(2 \pi \mathrm{f}_{\mathrm{c}}+\theta_{\mathrm{E}}\right)
\end{gathered}
$$

The synthetic signal sent by transmitter of SWIPT system is given by (1), where $P_{C_{k}}$ and $P_{C_{E}}$ denote information power and energy power respectively. $f_{c}$ is the carrier frequency. $\theta_{k}$ and $\theta_{E}$ denote information branch phase and energy branch phase respectively. $D_{k}(t)$ is information data and $x_{k}(t)$ is spread spectrum code.

Under the same frequency and time, each legitimate user terminal recovers its own signal according to the difference signal codes. The system user must have the same address code as the transmitter. The code is used for correlation detection of the received signal. Other signals cannot accomplish despread because they have different codes. Signal $\mathrm{B}$ can be seen as information interference in receiving process. Because the user terminal despreads the composite signal, signal $\mathrm{B}$ become a wide band and low power noise signal, and it will not affect the receiving information. If the Eavesdropper can complete receive a composite transmission signal $\mathrm{A}+\mathrm{B}$, because the power of $\mathrm{B}$ is higher than $\mathrm{A}$, the eavesdropper may be locked in the narrow signal B for in formation recovery. Without the knowledge of generating polynomial, eavesdropper cannot correctly despread to obtain true information. The physical layer encryption is achieved thereby. Moreover, the SWIPT CDMA transmission system can take advantage of the spreading gain and improve the signal-tonoise ratio. Even if signal is drowned in the noise, the receivers can correctly recover the whole information.

\section{OVSF CODE ADAPTIVE ALLOCATION SCHEME}

The CDMA system uses orthogonal variable spreading factor (OVSF) codes as channel code. The receiver needs to collect energy from the composite signal to process information. Subject to power consumption constraints, spreading factor or generating polynomial series cannot be too long. OVSF codes are limited. The restriction of orthogonality and the limitation of code resource will lead to code blocking. Code blocking reduces the utilization of code resource and makes the most codes obtaining by low rate call. For users, code assignment algorithm should cope with various rates calling fairly.

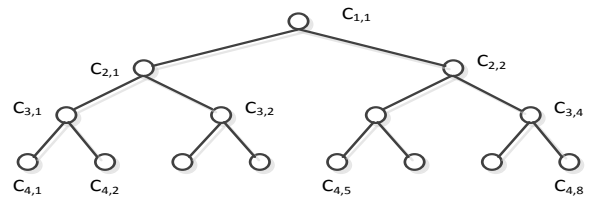

FIGURE III. THE OVSF CODE T REE ST RUCTURE (L=4)

$\mathrm{T}$ is OVSF-CDMA system tree head. $\mathrm{L}$ is the total number of layers, as shown in figure 3. The spreading factor of code tree is from 1 to $2^{\mathrm{L}-1}$, spreading factor of the l layer code is $2^{1-1}$. Code in the llayer is expressed by $C_{l, k}$, where $k$ is the located position in 1 layer, representing different codes. The data rate can be processed by code tree is $R \sim 2^{\mathrm{L}-1} \mathrm{R}$. Figure 3 shows the OVSF code tree of the spreading factor for $1 \sim 8$, can handle four different rates of $\mathrm{R}, 2 \mathrm{R}, 4 \mathrm{R}, 8 \mathrm{R}$. If the code a is derived by the upper code $b$, then $a$ is son code of $b$ and $b$ is father code of $\mathrm{a}$. Code assigned out is called busy code. Because his father code or son codes are busy codes, this code is called blocked code. The rest code is called vacant code. Sometimes the capacity of the system is able to support rate of new call, due to the codes scattered, more codes are blocked. It is unable to meet the new call and have to block the call. Th is blockage called code block. Code block reduces the utilization of code resource and causes the resource waste.

\section{A. Fixed Code Assignment (FCA) \& Adaptive Assignment}

According to the probability of each call rate, fixed code assignment divides the code tree into several non-overlapping areas. Each area supports one rate. The number of region is equal to the rate number. Under fixed code assignment, according to the probability of each rate call, code tree does not always accurately divide. The proportion of the probability of each rate call will change in the distribution process. Distribution area in advance cannot match with rate call probability. Although it has the merit of simple addressing, the fixed code assignment is not flexible. Other rate trees may be idle when one rate is blocking.

In the adaptive assignment, each tree can dynamically allocate to an arbitrary rate. When the rate tree has not vacant code, rate call has the right to use the adjacent blank tree. The blank tree is marked this rate. The merit of this method is that it can auto matically adapt to arrival rate calls and only needs to confirm the tree rate.

If the call needs a code of $l$ layer, it firstly confirms the tree rate. The largest number required search code tree is $T$. Adaptive assignment begins from the left of the code tree. It searchs the vacant code $C_{l, x}$. If there is a vacant code $C_{l, x}$, it is assigned to a new call. Otherwise a blank tree is distributed to the rate. The tree is $t,\{t \in T\}$. The $t$ tree codes are reserved for the same rate calls. Only the tree has one orthogonal code at least, reservation rate is effective. When all codes of one tree are released, the tree can be released and used to handle other rate.

\section{B. Modified Adaptive Assignment}

For high rate call with small spreading factor, such as $8 \mathrm{R}$, 4 layers tree can only accommodate 1 call, and is easily blocked. On the other hand, for the low rate call such as 1R, 
the same tree can accommodate 8 calls at the same time. The tree has more empty codes and it is not easy to be blocked.

This article puts forward a kind of modified adaptive assignment. The main idea is that a tree is not assigned one fixed rate and joints multi tree resources, realizing distribution according to need.

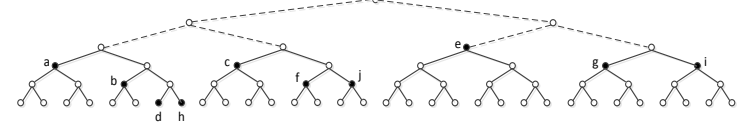

FIGURE IV. SCHEMATIC DIAGRAM OF THE MODIFIED ADAPTIVE ASSIGNMENT $\left(\mathrm{C}_{\mathrm{MAX}}=32\right)$

Assume four rates set $1 \mathrm{R}, 2 \mathrm{R}, 4 \mathrm{R}, 8 \mathrm{R}$, the coming call name is a, b, c, d, e, f, g, h, i and j. Rate calls are respectively $4 \mathrm{R}, 2 \mathrm{R}, 4 \mathrm{R}, 1 \mathrm{R}, 8 \mathrm{R}, 2 \mathrm{R}, 4 \mathrm{R}, 1 \mathrm{R}, 4 \mathrm{R}, 2 \mathrm{R}$. Total capacity is $32 \mathrm{R}$. The allocation process is shown in figure 4. Apparently under $32 \mathrm{R}$ total capacity, there is no block with modified adaptive assignment algorithm, while the calls are blocked twice in the adaptive assignment.

Among three algorithms, modified adaptive assignment has best throughput and minimal blocking rate, illustrating that modified adaptive assignment can deal with most calls under the same conditions. All code resources are occupied independently by all rate calls in fixed code assignment, and blocking calls can't share free resource of other areas. Adaptive assignment can adaptively allocate idle resources according to the arrival rate. But the degree of sharing resources is insufficient. Modified adaptive assignment shares code resources fully according to new rate calls. The idlest resource is allocated to the most needed call, so the throughput is largest. In a word, the throughput and minimal blocking rate performance of modified adaptive assignment is better than the other two algorith ms.

\section{RESUlts}

The call source is generated by a Poisson distribution with the average arrival rate $\lambda=1 \sim 16$ call nu mbers/unit time. The duration time obeys negative exponential distribution and the average length of time $1 / \mu=1$ is unit time. The maximum spreading factor is 8 , the spread factor range is $1 \sim 8$, and the number of the tree is $\mathrm{T}=4$. Using two different rates, request distribution $(\mathrm{R}, 2 \mathrm{R}, 4 \mathrm{R}, 8 \mathrm{R})=(0.40,0.40,0.10,0.10),(0.10$, $0.10,0.40,0.40)$. The total number of calls is 1000 time. We compare the throughput and blocking probability performance of fixed code assignment, adaptive assignment and modified adaptive assignment and the proposed rate distribution under two different conditions of velocity distribution.
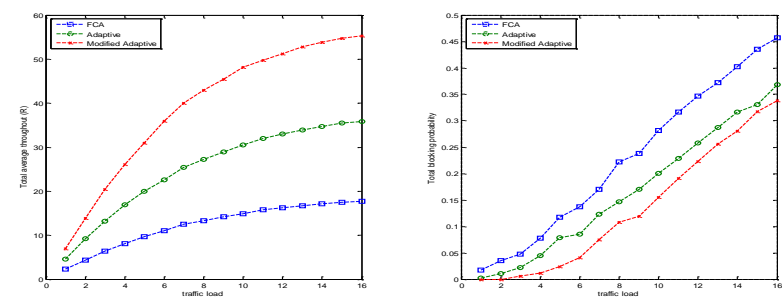

FIGURE V. THROUGHPUT AND BLOCKING PROBABILITY WITH RATE DIST RIBUTION [0.4 0.4 0.1 0.1$]$
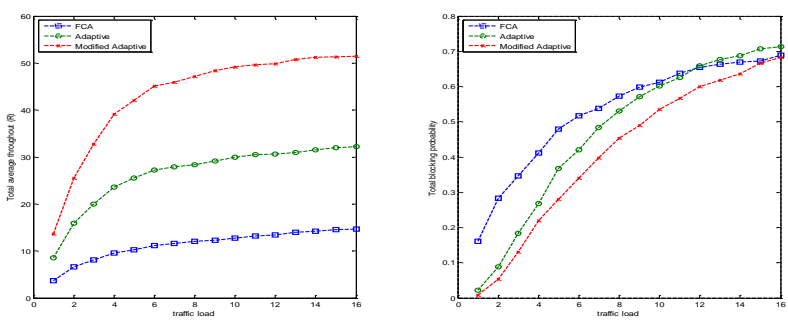

FIGURE VI. THROUGHPUT AND BLOCKINGPROBABILITY WITH RATE DIST RIBUTION [ $\left.\begin{array}{llll}0.1 & 0.1 & 0.4 & 0.4\end{array}\right]$

The throughput of adaptive assignment is better than fixed code assignment and blocking probability is below fixed code assignment. In Figure 5 and Figure 6, it can obviously be seen that modified adaptive assignment outperforms adaptive assignment. The throughput of modified adaptive assignment is higher than adaptive assignment and blocking probability is lower.

\section{CONCLUSION}

This paper addresses the important is sue of physical layer security SWIPT systems by combining the spread spectrum CDMA transmission techniques. CDMA can not only realize the wire less energy transmission, but also encrypt information transmission using spread spectrum codes. Generating polynomial of legitimate user keeps a secret to other legitimate users, so that the eavesdropper cannot correctly despread information and restore the content. In order to save energy, the length of spreading factor should not be too long, leading to a limited number of OVSF codes. Thus the rational allocation of the OVSF codes has an impact on the throughput of the system. The proposed model of modified adaptive assignment reduces the blocking probability of the system and the waiting time of the user. The simulation results also validate the effectiveness of modified adaptive assignment.

\section{ACKNOWLEDGMENTS}

This work is supported by Programs of Natural Science Foundation of China (No. 61201221, 61132004, 61275073), Key Laboratory of Specialty Fiber Optics and Optical Access Networks Shanghai University (SKLSFO2013-01) and Specialized Research Fund for the Doctoral Program of Higher Education of China (No.20123108120017).

\section{REFERENCES}

[1] Zhang R, Ho C K, MIMO broadcasting for simultaneous wireless informa-tion and power transfer. Wireless Communications, IEEE Transactions on, 12(5), pp. 1989-2001, 2013.

[2] Liu L, Zhang R, Chua K, Wireless information and power transfer: a dynamic power splitting approach. Communications, IEEE Transactions on, 61(9), pp. 3990-4001, 2013.

[3] Ng D, Lo E, Schober R, Wireless information and power transfer: energy efficiency optimization in OFDMA systems. Wireless Communications, IEEE Transactions on, pp. 6352-6370, 2013.

[4] Zhou X, Zhang R, Ho C, Wireless information and power transfer in multiuser OFDM systems. Wireless Communications, IEEE Transactions on, 13(4), pp. 2282-2294, 2014.

[5] Huang K, Larsson E G, Simult aneous information and power transfer for broadband wireless systems. Signal Processing, IEEE Transactions on, 61(23), pp. 5972-5986, 2013. 
[6] Ng D W K, Schober R, Resource allocation for secure communication in systems with wireless information and power transfer. Globecom Workshops (GC Wkshps), 2013 IEEE, pp. 1251-1257, 2013.

[7] Liu L, Zhang R, Chua K C, Secrecy wireless information and power transfer with MISO beamforming. Global Communications Conference (GLOBECOM), 2013 IEEE, pp. 1831-1836, 2013.

[8] Huang X, Li Q, Zhang Q, et al, Power allocation for secure OFDMA systems with wireless information and power transfer. Electronics Letters, 50(3), pp. 229-230, 2014. 